\title{
Analytical Method Selection for Drug Product Dissolution Testing
}

\author{
Qingxi Wang ${ }^{1,2}$, Decheng Ma ${ }^{1}$, and John P. Higgins ${ }^{1}$
}

e-mail:Qingxi_Wang@Merck.com

\begin{abstract}
This article provides an overview of analytical methods applied to the dissolution testing of various pharmaceutical products. The benefits and limitations of each method are discussed with respect to dynamic range, selectivity, extent of automation, efficiency, and robustness. This article presents useful guidance on analytical method selection for pharmaceutical development scientists and quality control personnel who are faced with the challenges of dissolution testing during the drug product life cycle.
\end{abstract}

\section{Introduction}

D issolution is a characterization test commonly used by the pharmaceutical industry to guide formulation design and control product quality. Often, it is a required performance test for solid dosage forms, transdermal patches, and suspensions. It is also the only test that measures the rate of in vitro drug release as a function of time, which can reflect either reproducibility of the product manufacturing process or, in limited cases, in vivo drug release. The objective of dissolution testing, in general, varies during the life cycle of a dosage form (1). The primary objective of dissolution testing at Phases 0 and $I$ is to establish the dissolution mechanism. During Phases II and III, the objective shifts to developing an understanding of the impact of key formulation/process parameters on dissolution and an in vitro-in vivo correlation (IVIVC). At product registration and beyond, the goal is to identify a quality control (QC) dissolution test method to verify process and product consistency. The continual evolution of objectives during the drug product life cycle may require different detection methods in order to have effective and efficient dissolution tests.

In general, analytical methods used for quantifying drug release in dissolution tests can be classified into four categories: spectrophotometric, chromatographic, mass spectrometric, and potentiometric. This article attempts to provide a comprehensive overview of these method categories, highlighting the advantages and limitations of each method as applied to dissolution testing. General considerations for method selection are discussed, focusing on five important analytical parameters: dynamic range, selectivity, extent of automation, efficiency, robustness, and compatibility with dissolution media. Finally, general guidance for method selection at different stages of drug product life cycle is presented.

\section{Direct Spectroscopic and Spectrophotometric Methods General Overview}

\section{UV/VIS Methods}

Direct UV/VIS spectrophotometric determination of absorbance has been the traditional analytical method for dissolution testing. A compound will exhibit absorption in the UV region if it contains one or more chromophores, such as aromatic nitro, azoxy, nitroso, carbonyl, or azo groups. The dynamic range of UV/VIS absorption is typically from 0.1 to 2 absorbance units for commercially available instruments. For quantitation of any given analytes, the desired absorption (A) should be $0.3-1.0$. As the sample concentration largely depends on the dose, the path length, which can be adjusted to achieve the desired absorption, is often the key parameter.

The development of commercial photodiodearray UV/VIS spectrometers paved the way for spectral deconvolution techniques that equipped UV spectrometers with the capability of handling multicomponent analysis or analysis in UV absorbing matrices (e.g., excipients). These techniques enable the components of a mixture to be determined simultaneously by optimizing the spectral fit between the sample spectrum and a weighted combination of the spectra of the individual components. For example, Anderson et al. (2) reported the dissolution testing of capsules and tablets using multi-component UV analysis, where interference from excipients presents a problem for direct UV analysis. Derivatives of the UV/VIS spectra present another solution for the elimination of analytical interferences. The features of the derivative UV 
Table 1. Advantages and Limitations of NIR, FTIR, and Raman Detection Methods

\begin{tabular}{|l|l|l|l|}
\hline Technique & $\begin{array}{c}\text { Suitable Concentration } \\
\text { Range }\end{array}$ & \multicolumn{1}{|c|}{ Key Advantages } & \multicolumn{1}{c|}{ Key Disadvantages } \\
\hline FTIR & 0.1 to $>100 \mathrm{mg} / \mathrm{mL}$ & $\begin{array}{l}\text { All organic compounds have suitable vibrational } \\
\text { bands, adequate sensitivity. }\end{array}$ & $\begin{array}{l}\text { Significant interference by aqueous } \\
\text { dissolution media. Complex instrumen- } \\
\text { tation. }\end{array}$ \\
\hline NIR & $5-1000 \mathrm{mg} / \mathrm{mL}$ & $\begin{array}{l}\text { Not affected by interference from aqueous } \\
\text { systems as FTIR. Simple instrumentation, rugged, } \\
\text { fiber-optic probes. All organic and most inorganic } \\
\text { compounds have suitable vibrational bands. }\end{array}$ & $\begin{array}{l}\text { Poor sensitivity. Instrument drifts; } \\
\text { requires calibration and maintenance. }\end{array}$ \\
\hline Raman & $10-1000 \mathrm{mg} / \mathrm{mL}$ & $\begin{array}{l}\text { Lowest effect of aqueous media. High degree of } \\
\text { molecular information. Suitable probes allow for } \\
\text { pure measurement of solution phase without } \\
\text { interference from the undissolved solids. }\end{array}$ & $\begin{array}{l}\text { Poor sensitivity. Complex, non-robust } \\
\text { instrumentation. }\end{array}$ \\
\hline
\end{tabular}

spectra allow the determination of one or more wavelengths where the drug compound of interest can be analyzed with zero or negligible absorption from the formulation matrix (3). Recent developments also include fiberoptic UV/VIS probes for in situ dissolution testing. An outstanding feature of these in situ methods is the capability to acquire continuous drug release in real time, hence providing information on critical kinetic behaviors of the active pharmaceutical ingredient (API) such as super-saturation, form conversion, intrinsic solubility, and also the entire kinetic process of the formulation dissolution (4). Though current fiber-optic UV/VIS methods suffer from inferior accuracy and variability due to the deposit of particles on the window of the UV/VIS detector, the demonstrated efficiency and wealth of information generated in real time make these in situ UV/VIS methods an excellent alternative to aid dissolution testing at early phase drug development. The direct flow injection analysis (FIA) system coupled with direct UV detection has been reported recently (5). This technique improves limits of quantitation and detection with minimum consumption of sample solution and can be applied to dissolution testing for controlled-release dosage forms.

\section{Fluorescence and Chemiluminescence Methods}

Fluorescence detection can be used if the compound is naturally fluorescent or can be made fluorescent via derivatization or photoirradiation. Fluorescence is the result of three sequential processes: excitation, intra-system crossing, and fluorescence emission. The difference in energy or wavelength of excitation and emission is referred to as the Stokes Shift, and is fundamental to the sensitivity of the fluorescence technique since it allows the detection of emitted photons against a low background, isolated from the excitation photons. The fluorescent intensity $\Phi_{L}$ is directly proportional to the analyte concentration c and the incident radiant power $\Phi_{0}$ (6):

$$
\Phi_{L}=k^{\prime} \Phi_{0} C
$$

where $\mathrm{k}^{\prime}$ is a constant depending on the species and the environment. As the sample absorbance goes beyond 0.05 in a $1-\mathrm{cm}$ path length, the relationship becomes nonlinear. In general, fluorescence detection offers superior sensitivity and selectivity compared to that provided by UV detection. It can be particularly useful for the dissolution testing of fluorescence-bearing drug products in complex formulation matrices. Dissolution testing with both offline and online fluorescence detection has been reported $(7,8)$.

Chemiluminescence $(\mathrm{CL})$ detection may be employed for dissolution analysis when the analyte lacks a chromophore. The $C L$ technique is based on the principle that part of the chemical energy released in certain reactions is used to produce excited species (9). The CL detector is typically 10 times more sensitive than the fluorescence detector, and the limit of detection (LOD) is $1 \mathrm{pmol}$ for $\mathrm{CL}$ and $10 \mathrm{pmol}$ for fluorescence detector, respectively. Very few applications of $\mathrm{CL}$ in the dissolution testing of pharmaceuticals have been published (10).

\section{IR/Raman Methods}

Fourier transform infrared (FTIR), near infrared (NIR), and Raman spectroscopic techniques have not been reported as the direct detection method of choice for compendial dissolution testing since they often require high concentration of analytes. However, they present another cluster of potentially powerful online spectrophotometric tools for dissolution testing. The advantages, limitations, and suitable sample concentration ranges for FTIR, NIR, and Raman techniques are summarized in Table 1.

Similar to UV/VIS, Beer's law provides the basis for quantitative analysis with IR detection methods. However, nonlinearities and band overlap can pose great challenges. Dissolution of ordinary tablets cannot usually be analyzed by FTIR absorption spectroscopy due to the high absorptivity of water. In addition, the long path length will lead to 


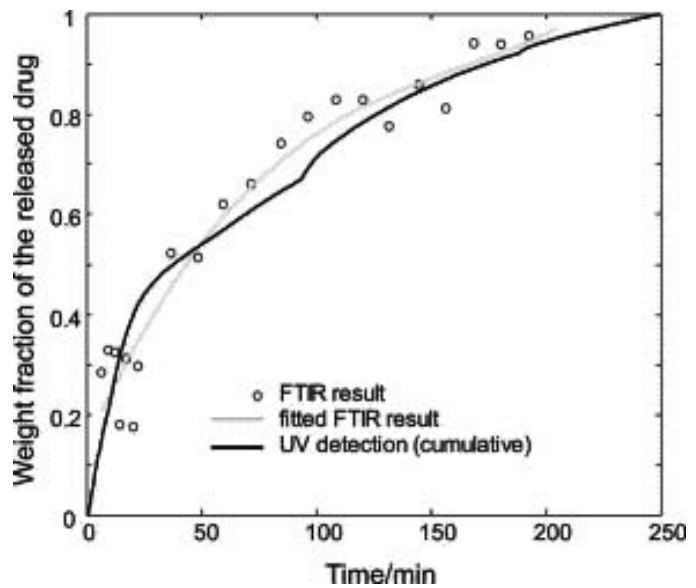

Figure 1. Dissolution Profiles of Nicotinamide Tablets from ATR-IR Imaging and Direct UV Detection

complete absorption of the incident IR light. Despite its limitations, FTIR imaging in attenuated total reflection (ATR) mode in combination with traditional dissolution detection methods such as UV/VIS has been proven useful in allowing simultaneous measurements of the distribution of different components in the tablet, for example, drug and polymer as a function of time. These imaging measurements were carried out in a combined compaction and flow-through cell that was linked to a UV detector to quantify the amount of dissolved drug. This combination provides quantitative information regarding changes in both the tablet and the liquid phase. This setup was successfully used by van der Weerd et al. (11) to investigate the dissolution behavior of nicotinamide controlled-release tablets. The comparable drug release profiles were obtained by UV and FTIR imaging simultaneously (Figure 1).

NIR spectroscopy has some similarities to both UV/VIS and mid-IR. The majority of the absorption bands in the NIR region are related to combinations and overtones of fundamental bands that usually correspond to hydrogen-stretching vibrations such as $\mathrm{C}-\mathrm{H}, \mathrm{O}-\mathrm{H}$, and $\mathrm{N}-\mathrm{H}$ (12). The NIR spectrum has characteristics of low intensity, highly overlapping, and broad bands. Thus, it is difficult to use NIR to measure samples with low concentrations due to its low sensitivity. Nevertheless, the quantitative NIR spectral information can still be successfully extracted with multivariate calibration techniques such as partial-least-square (PLS) regression for various applications including dissolution testing. As an example, a feasibility study demonstrated that the dissolution of immediaterelease stressed tablets can be correlated with their NIR spectra using percent API dissolved after 20 minutes (\% 20 $\mathrm{min}$ ) as the single correlation parameter (13).

Raman spectroscopy is a molecular scattering technique that measures the wavelength and intensity of inelastically scattered light from molecules. Like FTIR, Raman is useful for identification and quantitation of both organic and inorganic compounds and functional groups. The two techniques are complementary:FTIR is ideally suited to analyze polar compounds, while Raman spectroscopy is better for analyzing non-polar compounds. Raman spectroscopy has been developed as the detection technique for lincomycin $\mathrm{HCl}$ capsules (14).

\section{Mass Spectrometric Methods}

Mass spectrometry (MS) is a spectroscopic tool primarily concerned with the separation of gas phase ions according to their mass-to-charge ratios $(\mathrm{m} / \mathrm{z})$. The major components of a mass spectrometer include the sample inlet system, ion source, mass analyzer, and detector. Sample introduction usually involves coupling the mass spectrometer directly to an HPLC, gas chromatograph (GC), or CE; hence, the sample components are separated sequentially prior to entering the mass spectrometer for analysis (15). Many ionization methods are available, and each has its own advantages and disadvantages (16). Commonly used ionization methods include electrospray ionization (ESI), atmosphere pressure chemical ionization (APCl), electron impact (EI), chemical ionization (Cl), matrix assisted laser desorption ionization (MALDI), fast atom bombardment (FAB), field desorption/field ionization (FD/FI), and thermospray ionization (TSP). ESI and APCl are widely used for LC/MS. Selected ion monitoring and tandem mass spectrometry enable the mass detector to analyze specific compounds in a complex mixture. Therefore, it has been widely used for the rapid screening of drug compounds in biological fluids (17-20). The main advantage of MS is its capability for analyzing both chromophoric and non-chromophoric drug compounds with superior selectivity and sensitivity. For example, a tandem mass spectrometric (MS/MS) procedure with turbo ion spray ionization was developed for the dissolution testing of alendronate sodium tablets where the active pharmaceutical ingredient has no UV-absorbing chromophores (21).

The use of the mass spectrometric technique in routine dissolution testing for formulation development support and drug product quality control has not been recognized due to the following limitations of mass spectrometric instrumentation:(1) The mass detector is still relatively expensive for routine quality control. (2) Few well-defined analytical procedures fit current GMP requirements. For instance, due to the suppression of ionization from the matrix (e.g., excipient, dissolution medium, and sample diluent), quantitation is often times limited to the use of internal standards. (3) Method ruggedness and robustness issues associated with mass spectrometry are the result of an unstable ionization source and/or significant interference from the formulation excipients and dissolution media.

\section{Potentiometric Titration Methods}

In the early days of dissolution testing, potentiometric titration techniques were used to determine the dissolution rate of acidic and basic drugs (22). Potentiometric methods are based upon measurements of the potential of electrochemical cells in the absence of appreciable currents. Recent developments use ion-selective membrane electrodes to obtain ion concentrations directly. lon-selective electrodes are relatively free from interference and provide 


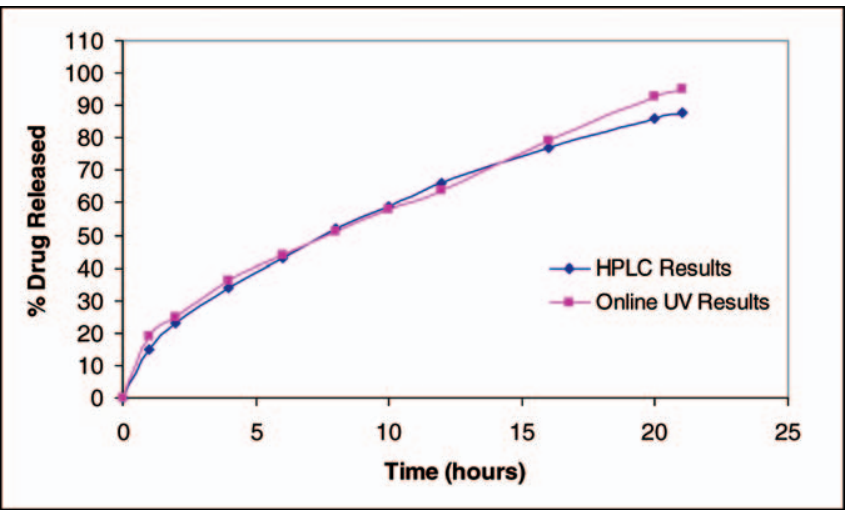

Figure 2. Dissolution Profiles of a Controlled-Release Tablet Formulation by Online UV Detection versus HPLC

a rapid and convenient means for quantitative analysis. Potentiometric methods have not been used recently in routine dissolution testing of pharmaceuticals due to the lack of adequate sensitivity, specificity, and accuracy.

\section{Method Benefits and Limitations}

Direct methods are simple, fast, direct, and as with fiberoptic probes, can provide data in real time. With these features, they are well suited for online monitoring of the dissolution process. As an example, Figure 2 shows dissolution profiles of a controlled-release tablet formulation analyzed by online UV detection. The data obtained compared well with the HPLC method. Fluorescence and chemiluminescence detection methods provide superior selectivity and can be applied to dissolution testing for complex formulations. IR imaging has demonstrated its utility in monitoring drug distribution in the solid dosage form during dissolution and, therefore, can be a valuable tool for understanding the dissolution mechanism. The disadvantages of these methods should be noted as well. Clearly, all of the methods lack a wide dynamic range (1-2 orders of magnitude at best), which often makes it impossible to support dissolution testing of formulations having the wide range of doses common to early drug development. Analytical interference from other components in the formulations, such as the formulation excipients, active pharmaceutical ingredients for multi-component dosage forms, and dissolution media, presents another issue for direct UV detection. When two or more active ingredients are included in the dosage form, spectrophotometric methods often cannot be used, primarily due to cross-interference of active pharmaceutical ingredients. Though recently developed UV/VIS spectrometers can perform multi-component analyses for some combinational dosage forms, additional method development including investigation of data treatment is needed for them to be acceptable for routine dissolution testing. The vibrational spectroscopic methods (IR and Raman) suffer from sensitivity limitations
(Table 1). Furthermore, quantitation can be challenging without proper calibration and standard considerations.

\section{Separation-Based Methods General overview}

High Performance Liquid Chromatography (HPLC)

The HPLC separation process involves the partition of analytes between a stationary phase (packed in a column) and a mobile phase. The difference in mobility results in the separation of different components as they travel through the stationary phase, which is identified by retention time $\left(t_{R}\right)$ on the chromatogram. The column efficiency can be measured as the number of theoretical plates in a column $(N)$ :

$$
N=16\left(\frac{t_{R}}{W^{2}}\right)=5.54\left(\frac{t_{R}}{W_{1 / 2}}\right)^{2}
$$

where $W$ and $W_{1 / 2}$ are the peak width at the baseline and at the half height, respectively.

A large value for theoretical plates $(N)$ indicates an efficient column, which allows sample molecules to migrate in a narrow band (small $W$ or $W_{1 / 2}$ ) with minimal dispersion (23). Therefore, the theoretical plate $(N)$ also indicates the sharpness of a peak. The chromatographic peak area or peak height is used for quantitative analysis. It is expressed in millivolts $(\mathrm{mV})$, typically in the range of $0.1-1000$ with current data collection systems.

The UV detector is the most commonly used in HPLC methods for dissolution testing. However, other detector choices allow pharmaceutical scientists to use HPLC for dissolution testing of various drug products that otherwise could not be done with UV detection. An electrochemical detector coupled with LC is the fastest growing technique for biomedical analysis and is also used in the dissolution testing of pharmaceutical dosage forms (24). It offers the advantages of low detection limits (sub-picomole range) and selectivity. The selectivity of electrochemical detectors is pronounced when analyzing electrochemically active drugs in highly complex formulations. Thermal conductivity and refractive index detectors may also be used for the detection of both chromophoric and non-chromophoric compounds. Though suffering from inadequate sensitivity and ruggedness, these methods can still be used in the dissolution testing of high potency dosage forms in simple aqueous media (dissolution media containing surfactants can be problematic due to analytical interference). The application of an MS detector coupled with LC has been reported in dissolution testing of several drug products. For example, an LC/MS method with atmospheric pressure chemical ionization (APCl) was developed for a dissolution assay of melatonin from tablet and capsule dosage forms and nifedipine controlled-release formulations to probe in vivo-in vitro correlation (IVIVC) $(25,26)$. Recently, HPLC with evaporative light scattering detection (ELSD) has been particularly useful for the analysis of dissolution samples lacking chromophores (27). Major advantages for ELSD 
include high sensitivity and stable baselines that are suitable for gradient methods (28). Whelan et al. (29) have developed an HPLC-ELSD method that detected ibuprofen and hydroxypropylmethyl cellulose (HPMC) simultaneously. The limits of quantitation (LOQ) for ibuprofen and HPMC are 0.07 and $0.03 \mathrm{mg} / \mathrm{mL}$, respectively. Since HPMC is widely used as a drug-release modifier, this method provides an approach to better understand the dissolution mechanism of controlled- or modified-release formulations by monitoring the release of API and HPMC simultaneously. With dissolution testing of pharmaceutical products often requiring the testing of analytes in complex matrices, the use of ELSD and other non-selective detection methods is still limited.

\section{Capillary Electrophoresis (CE)}

With ionic drug compounds that often can not be retained on traditional reversed phase columns, CE can be an excellent alternative separation technique to HPLC (30). Compared with HPLC, the instrumentation for CE is simpler in design:two separate buffer reservoirs containing an anode and cathode electrode, respectively, are connected with a buffer-filled, narrow-bore capillary (normally 25-100 $\mu \mathrm{m}$ ID). When a voltage (up to $30 \mathrm{kV}$ ) is applied across the electrodes, the anions and cations in the solution within the capillary move toward the electrode of opposite charge. The principles of CE separation refer to the differential movement or migration of ions by attraction or repulsion in the electric field. Therefore, the separation by electrophoresis relies on the differences in the speed of migration of ions or solutes, which is expressed as

$$
\vec{v}_{e}=\vec{\mu}_{e} \cdot \vec{E}
$$

where $v_{\mathrm{e}}$ is electrophoresis migration velocity, $\mu_{\mathrm{e}}$ is electrophoretic mobility, and $E$ is the electric field strength (31). Electrophoretic mobility is a parameter that indicates how rapidly a given ion or solute can move through a given medium. At steady state, $\mu_{\mathrm{e}}$ is a constant and can be expressed as

$$
\mu_{e}=\frac{q}{6 \pi \eta r}
$$

where $\eta$ is the solution viscosity, and $q$ and $r$ are the charge and radius of the ion, respectively. It shows that in a given medium, the electrophoretic mobility (migration speed) is determined by the charge-to-size ratio of the analyte ions. The other factor affecting the migration speed is the electro osmotic flow (EOF). This is the bulk flow of liquid due to the effect of the electric field on the counterions adjacent to the negatively charged capillary wall. Although CE can be an excellent alternative to HPLC for ionic drug molecules, its limit of detection (LOD) is usually about 50 times poorer than that of HPLC. This is partially due to its much smaller path length (e.g., $50 \mu \mathrm{m})$ compared with that of HPLC (e.g., $10 \mathrm{~mm}$ ). There are continuous improvements in instrumental design to narrow this detection gap (32).

The applications of CE methods in dissolution testing have been reported. An easy and accurate CE method with UV and amperometric detection was claimed for the disso- lution testing of melatonin drug product $(33,34)$. Flow-injection CE was recently developed by Karlberg's (35) and Fang's groups (36) independently. Subsequently, this technique was applied to the dissolution testing of sulfatrim tablets containing trithoprin and sulfamethoxazole; meanwhile, the emphasis on the need to improve the ruggedness of the instrumentation and methods, synchronize the different means of liquid propulsion, and develop the miniaturized systems were noted (37). With flow-injection CE, the dissolution samples can be automatically and consecutively injected into the separation capillary without current interruption, hence greatly improving the sample throughputs (at least three times) over conventional CE methods. Finally, CE systems with ion suppression have been developed, which may allow use of the CE method for dissolution testing in the presence of buffers and ionic excipients.

\section{Method Benefits and Limitations}

HPLC and CE methods render the following advantages: wide dynamic linear range, selectivity via separation, and superior sensitivity. These features have been used to solve a variety of analytical problems encountered during dissolution testing of complex drug delivery systems. HPLC methods typically have a linear range of up to 3-4 orders of magnitude. Such a wide dynamic range often allows pharmaceutical researchers to conduct the dissolution testing of formulation doses ranging from 0.1 to $200 \mathrm{mg}$ with a single HPLC method. HPLC methods also afford superior sensitivity over direct spectrophotometric methods and are often used for dissolution testing of drug products with very low potencies. As an example, Swartz et al. (38) reported the dissolution testing of oral contraceptives containing $1 \mathrm{mg}$ of norethindrone and $0.05 \mathrm{mg}$ ethinylestradiol, where sensitive HPLC was used to analyze sample solutions with concentrations of $1.7 \mu \mathrm{g} / \mathrm{mL}$ and $0.06 \mu \mathrm{g} / \mathrm{mL}$, respectively, for both active components. Transdermal dosage forms (patches, creams, and ointments) are characteristic of very low drug dose (as low as pg/mL). The dissolution samples for these dosage forms have been best analyzed by HPLC methods (39). Another advantage of HPLC over spectrophotometric methods lies in its separation capability. Through chromatographic separations, the analytes of interest can be detected and quantified in the presence of degradation products, excipients (including antioxidants and preservatives), and surfactant dissolution media. Yang et al. (40) used ion-pair HPLC to monitor the dissolution of pentamdidine from EVA sustained-release film where polymeric matrices could create significant bias if a spectrophotometric method were used. Mariappan et al. (41) reported the simultaneous dissolution monitoring of rifampicin, isoniazid, and pyrazinamide using reversed phase HPLC. The method worked well for analysis of all three drugs in the presence of excipients and degradation products. However, because of the need for separation, neither HPLC nor CE methods are as efficient as direct spectrophotometric methods. The analysis time often cannot be synchronized with pre-determined dissolution sampling timepoints, which may limit the extent of automation. 
Table 2. Compatibility of Analytical Methods with Commonly Used Dissolution Methods

\begin{tabular}{|c|c|c|c|}
\hline \multirow[t]{2}{*}{ Dissolution Media } & \multicolumn{3}{|c|}{ Detection Method Choice } \\
\hline & Chromatographic & Spectrophotometric & Mass Spectrometric \\
\hline Water & No interference & No interference & No interference \\
\hline Buffers (pH 4.5,6.8) & No interference & $\begin{array}{l}\text { Depending on detection wavelength, } \\
\text { refer to buffer UV cut-off }\end{array}$ & $\begin{array}{l}\text { Significant interference due to } \\
\text { ion suppression }\end{array}$ \\
\hline$\underline{\mathrm{HCl}(0.01 \mathrm{~N}, 0.1 \mathrm{~N})}$ & $\begin{array}{l}\text { Occasional incompatibility with } \\
\text { mobile phase. Sample dilution may } \\
\text { be needed. }\end{array}$ & No interference & $\begin{array}{l}\text { Significant interference due to } \\
\text { ion suppression }\end{array}$ \\
\hline Tween $80(0.1-5 \%)$ & $\begin{array}{l}\text { Significant chromatographic inter- } \\
\text { ference esp. at high concentrations. }\end{array}$ & Significant interference & Significant interference \\
\hline$\underline{\text { SLS \& C-Tab }(0.1-5 \%)}$ & $\begin{array}{l}\text { Occasional incompatibility with } \\
\text { mobile phase. Sample dilution may } \\
\text { be needed. No analytical interfer- } \\
\text { ence. }\end{array}$ & $\begin{array}{l}\text { Significant interference depending on } \\
\text { detection wavelength and concentra- } \\
\text { tion. }\end{array}$ & Significant interference \\
\hline
\end{tabular}

\section{Analytical Method Selection for Dissolution Testing During Drug Product Life Cycle \\ Method selection based on drug chemical properties}

The selection of a suitable analytical method for dissolution testing is governed primarily by the chemical properties of the drug compound being analyzed. The properties include but are not limited to the presence of functional groups (chromophores), ionizability, and polarity and should be examined before method development is initiated. The most commonly used detection is UV/VIS for drug compounds with one or more chromophoric functional groups. The detection techniques with higher selectivity and sensitivity such as electrochemical, fluorescence, and mass spectrometric detection should be considered when direct or separation-based UV detection is deemed unsuitable. Occasionally, chemical modification or derivatization may be used for analysis of a drug compound lacking any chromophores.

\section{Method selection based on compatibility with dissolution media}

The compatibility of analytical methods with dissolution media cannot be ignored and is often a key factor to consider during method selection. The media used in dissolution methods for quality control are limited and regulated to simulate the physiological fluids. For aqueous soluble drug substances, water, acids ( 0.01 or $0.1 \mathrm{~N} \mathrm{HCl}$ ), and buffers $(\mathrm{pH} \leq 7.0)$ are commonly used dissolution media. With poorly soluble drug substances, surfactants are added to enhance solubility or wettability. The commonly used dissolution media and their compatibility with selective detection methods are illustrated in Table 2. Non-selective methods (RI, ELSD, etc.) are not discussed here as they are incompatible with most dissolution media. Aqueous media are often compatible with both spectrophotometric and separationbased methods, though sample dilution may occasionally be needed prior to chromatographic analysis. Surfactant media such as Tween 80 and sodium lauryl sulfate (SLS) often present significant analytical interference for spectrophotometric methods. Tween 80 with high purity is commercially available from selected suppliers and has been found to pose no significant analytical interference with HPLC methods, even with concentrations of up to $5 \%(\mathrm{w} / \mathrm{w})$. Sodium lauryl sulfate (SLS) does not interfere with direct UV/VIS and HPLC methods at a wavelength above $240 \mathrm{~nm}$ and, therefore, is commonly used in dissolution media in lieu of Tween 80 . Dissolution media with added enzymes are occasionally used for IVIVC or dissolution of hard gelatin capsule dosage forms to overcome capsule cross-linking (pellicle formation) (42). Similarly, enzymes create significant interference with drugs of interest. To navigate the problems associated with compatibility of detection methods and dissolution media, the following tactics may be considered:

(1) The use of separation-based detection methods. HPLC methods should be considered as the starting point to eliminate the interference from surfactants.

(2) The use of appropriate sample treatment. The dissolution samples may be diluted with salt, buffer, HPLC, or CE mobile phase, as the dissolution testing procedure defined in USP frequently calls for the sample to be diluted with mobile phase and salt/buffer solutions to avoid incompatibility of the dissolution medium and mobile phase and to break up micelles.

(3) The use of selective detection methods. Selective detection methods such as fluorescence, electrochemical, and mass detection can be considered to analyze drug compounds in highly complex dissolution samples that would pose significant analytical interference with direct 


\begin{tabular}{|c|c|c|c|c|c|c|}
\hline \multirow[t]{2}{*}{ Drug Product Life Cycle } & \multicolumn{5}{|c|}{ Detection Method Characteristics } & \multirow[t]{2}{*}{ Recommended method } \\
\hline & $\frac{\text { Dynamic }}{\text { Range }}$ & Selectivity & $\underline{\text { Extent of }}$ & Efficiency & $\underline{\text { Robustness }}$ & \\
\hline $\begin{array}{l}\text { Preclinical-Phase II } \\
\text { Formulation design } \\
\text { Process scale-up } \\
\text { Dissolution mechanism }\end{array}$ & +++ & ++ & - & + & - & $\begin{array}{l}\text { HPLC/CE methods preferred to } \\
\text { support wide dose range. } \\
\text { Direct UV/NIR methods with fiber- } \\
\text { optic probes are used for dissolu- } \\
\text { tion mechanism }\end{array}$ \\
\hline $\begin{array}{l}\text { Phase III } \\
\text { Long term stability } \\
\text { Discriminating evaluation } \\
\text { IVIVC (if needed) }\end{array}$ & + & ++ & +++ & +++ & ++ & $\begin{array}{l}\text { Direct UV/VIS method preferred } \\
\text { for efficiency and automation } \\
\text { Direct fluorescence and HPLC } \\
\text { methods can be used if selectivity } \\
\text { is needed due to complex formu- } \\
\text { lation and/or surfactant dissolu- } \\
\text { tion media }\end{array}$ \\
\hline $\begin{array}{l}\text { Registration \& Marketing } \\
\text { Quality control } \\
\text { Bioavailability } \\
\text { Post-Approval Change }\end{array}$ & - & ++ & ++ & ++ & +++ & $\begin{array}{l}\text { Direct UV/VIS method are } \\
\text { preferred. } \\
\text { HPLC methods are used if separa- } \\
\text { tion is needed for selectivity }\end{array}$ \\
\hline
\end{tabular}

Level of importance: - not considered; + low; ++ medium; +++ high

UV/VIS detection and even with separation-based methods.

(4) The use of surfactants with low concentration and/or high purity. The dissolution medium with the lowest concentration of surfactants that affords satisfactory profiles should always be adopted to minimize analytical interference and incompatibility with the mobile phase. This approach also complies with global regulatory guidance and expectations.

\section{Method selection based on drug product life cycle}

A variety of analytical techniques can be amenable to dissolution testing. However, pharmaceutical researchers should consider five method characteristics when selecting the optimal analytical method for dissolution testing: dynamic range, selectivity, automation, efficiency, and robustness. Based on the best knowledge of the authors, the relative importance of these characteristics and how they guide method selection at different stages of drug product life cycle is summarized in Table 3.

At early stages of drug development, developmental dosage forms with a wide range of potency (e.g., 0.1-200 $\mathrm{mg}$ ) are often used in clinical studies. Automation and robustness are not considered at this stage due to the lack of experience with the methods or the need for multi-laboratory method transfer. Therefore, an HPLC or CE method is preferred as it provides not only a wide dynamic range but also the desired selectivity needed for complex dosage forms and a surfactant dissolution media. However, spectroscopic techniques such as fiber-optic UV, NIR, and Raman with fiber-optic probes should be used to probe API intrinsic solubility, kinetics of the dissolution process, and the dissolution mechanism, wherever practical. In Phase III drug development, numerous dissolution samples are tested to support long-term stability studies, production of pilot scale batches, and possibly IVIVC. Efficiency and automation are desired. Dynamic range becomes less important as a drug development program progresses into Phase III when the distinct market doses are selected. Therefore, a UV/VIS detection method should be used as it clearly demonstrates the advantages as simple, fast, cost effective, and easily interfaced with current dissolution apparatus to achieve automation. In some cases, the analytical interference with surfactant media and/or formulation matrix prohibits the use of UV/VIS methods. Instead, selective methods (e.g., fluorescence detection) or HPLC methods are used. After the drug product launches onto the market, the dissolution method will be transferred to quality control laboratories. Method robustness, ease of use, and efficiency are considered important. In addition, instrument availability and available training at quality control sites should be carefully evaluated when selecting the detection methods. As most quality control laboratories are commonly equipped with 
HPLC and UV, detection methods such as mass spectrometry, NIR, or Raman are generally not considered at this late stage of the drug product life-cycle.

\section{Conclusion}

Detection methods and instrument technology have progressed to the point that pharmaceutical scientists are granted tremendous flexibility in selecting an optimal detection method for dissolution testing. Novel uses will continue to be discovered as pharmaceutical researchers explore ways of obtaining real-time dissolution results with satisfactory accuracy, precision, and reproducibility. Online analysis and automation are the key factors for detection method choice as the pharmaceutical industry drives for efficiency and productivity. As formulation development trends toward more complex drug delivery systems, separation-based detection and mass detection methods will be used more often in dissolution testing due to their distinct advantages over spectrophotometric methods.

\section{References}

1. Brown, C. K.; Chokshi, H. P.; Nickerson, B.; Reed, R. A.; Rohrs, B. R.; Shah, P. A. Pharm. Tech. 2004, 28, 56-65.

2. Anderson, N. H.; Johnston, D.; Vojvodic, P. R. J. Pharm. Biomed. Anal. 1990, 8 (8-12) 987-989.

3. Wang L.; Asgharnejad, M. J. Pharm. Biomed. Anal. 2000, 21, 1243-1248.

4. Cho, J. H.; Gemperline, P. J.; Salt, A.; Walker, D. S. Anal. Chem. 1995, 67 (17), 2858-2863.

5. Gemperline, P. J.; Cho, J. H.; Baker, B.; Batchelor, B.; Walker, D. S. Anal. Chim. Acta 1997, 345 (1-3) 155-159.

6. Ingle, J. D.; Crouch, S. R. Spectrochemical Analysis; Prentice-Hall: New Jersey, 1988; $p 338$.

7. Abdel-Moety, E. M.; Moustafa, A. A.; Ahmad, A. K. S.; ElGendy, A. E. Sci. Pharm. 1987, 55 (4), 259-265.

8. Rogers, P.; Hailey, P. A.; Johnson, G. A.; Dight, V. A.; Read, C.; Shingler, A.; Savage, P.; Roche, T.; Mondry, J. Lab. Rob. Autom. 1999, 12, 12-22.

9. Ingle, J. D.; Crouch, S. R. Spectrochemical Analysis; Prentice-Hall: New Jersey, 1988; pp 478-480.

10. Solich, P.; Polydorou, C. K.; Koupparis, M. A.; Efstathiou, C. E. Anal. Chim. Acta 2001, 438, 131-136.

11. van der Weerd, J.; Kazarian, S. G. J. Control. Release 2004, 98, 295-305.

12. Ku, M. S.; Chung, H. Appl. Spectrosc. 1999, 53 (5), 557-564.

13. Kuny, T.; Schatz, C.; Ulmschneider, M.; Marrer, S.; Leuenberger, H. Dissolution Technol. 2003, 10 (1), 22-28.

14. Lincomycin $\mathrm{HCl}$ Capsules. United States Pharmacopeia and National Formulary USP 29-NF 24; United States Pharmacopeial Convention, Inc.: Rockville, MD, 2005.

15. Ball, D. W. Spectrosc. 2001, 16 (11), 32-34.

16. Mass Spectrometry $\langle 736\rangle$. United States Pharmacopeia and National Formulary USP 28-NF 23; United States Pharmacopeial Convention, Inc.: Rockville, MD, 2004.

17. Karlberg, B.; Kuba, P. J. Flow Injection Anal. 2000, 17 (1), 5-9.
18. Niessen, W.M.A. Liquid Chromatography-Mass Spectrometry, 2nd ed.; Marcel Dekker: New York, 1998; p 405.

19. Voyksner, R. D. Combining Liquid Chromatography with Electrospray Mass Spectrometry. In Electrospray lonization Mass Spectrometry: Fundamentals, Instrumentation, and Applications; Cole, R. B., Ed.; Wiley Interscience: New York, 1997; p 323.

20. Henion, J.; Prosser, S. J.; Corso, T. N.; Schultz, G. A. Am. Pharm. Rev. 2000, 3, 19.

21. Wang, Q.; Zhao, J.-G.; Ballard, J. M.; Shambor, S. M.; Tsai, E. W.; Ip, D. Application of Tandem Mass Spectrometry to FOSAMAX ${ }^{\circledR}$, dissolution testing. Mass Frontier Symposium, Purdue University, October 10, 1999.

22. Shah, A. C. J. Pharm. Sci. 1971, 60, 1564-1567.

23. Cunico, R. L.; Gooding, K. M.;Wehr,T. Basic HPLC and CE of Biomolecules; Bay Bioanalytical Laboratory: Richmond, CA, 1998; pp 14-25.

24. Begona, B. M.; Alonso, R. M.; Jimenez, R. M. J. Liq. Chromatogr. Relat. Technol. 1996, 19, 179-186.

25. Xie, F.-M.; Wong, P.; Yoshioka, K.; Cooks, R. G.; Kissinger, P. T. J. Liq. Chromatogr. Relat. Technol. 1998, 21 (9), 1273-1282.

26. Natishan, T. K. J. Liq. Chromatogr. Relat. Technol. 2004, 27, 1237-1316.

27. Allgeier, M. C.; Nussbaum, M. A.; Risley, D. S. LCGC North America, 2003, 21 (4), 376-381.

28. Meehan, E.; Saunders, G.; Bullock, S. LCGC North America 2003, June Suppl. S., 48-49.

29. Whelan, M. R.; Ford, J. L.; Powell, M. W. J. Pharm. Biomed. Anal. 2002, 30, 1355-1359.

30. Schug, B. S.; Benddel, E.;Wonnemann; M.;Wolf, D. Eur.J. Clin. Pharmacol. 2002, 58 (2), 119-125.

31. Grossman, P. D.; Colburn, J. C. Capillary Electrophoresis Theory and Practice; Academic Press, Inc.: San Diego, CA, 1992; pp 14-19, 111-120, 304-306.

32. Frazier, R. A.; Ames, J. M.; Nursten, H. E. Capillary Electrophoresis for Food Analysis: Method Development; Royal Society of Chemistry: London, 2001; pp 1-6.

33. Cartoni, G. P.; Coccioli, F.; Jasionowska, R.; Masci, M. Chromatographia 2000, 52 (9/10), 603-606.

34. You, T.; Liu, Z.; Yang, X.;Wang, E. Talanta 1999, 49, 517.

35. Kuban, P.; Engstrom, A.; Olsson, J. C.; Thorsen, G.; Tryzell, R.; Karlberg, B. Anal. Chim. Acta 1997, 337, 117.

36. Fang, Z. L.; Liu, Z. S.; Shen, Q. Anal. Chim. Acta 1997, 346, 135.

37. Fang, Z. L.; Chen, H. W. Anal. Chim. Acta 1998, 376, 209-220.

38. Swartz, M.; Neitring, I. Am. Lab. 1990, 22 (40), 24-26.

39. Li, J. B.; Rahm, P. C. Pharm. Tech. 1993, 17 (7), 44-54.

40. Yang, C.-Z. Zhongguo Yaoxue Zazhi (Beijing, China) $1992,27(1), 21-22$.

41. Mariappan, T. T.; Singh, B.; Singh, S. Pharm. Pharmacol. Comm. 2000, 6 (8), 345-349.

42. Murphy, K. S.; Reisch, R. G. J.; Fawzi, M. B. Pharm. Tech. 1989, 13, 53-58. 